\title{
Perfiles de enseñanza según formas de interacción comunicativa en el aula. Estudio de caso con seis profesores de ciencias naturales
}

\author{
Teaching Profiles According to Ways of Assuming Communicative \\ Interaction in the Classroom. A Case Study with Six Science Teachers
}

Perfis de ensino segundo as formas de interação comunicativa na sala
de aula. Estudo de caso com seis professores de ciências natirais

Ángela Camargo-Uribe ${ }^{1}$ Christian Hederich-Martínez ${ }^{2}$

Resumen

El artículo reporta un proceso investigativo llevado a cabo con el propósito de identificar perfiles de enseñanza a partir del análisis de formas de interacción comunicativa en el aula. Participaron en el estudio seis profesores de ciencias naturales de dos colegios oficiales de Bogotá, Colombia, que ejercen la docencia en los grados sexto a octavo de educación básica. Se grabaron secuencias de tres clases por cada profesor, cada una de las cuales se analizó luego para identificar las actividades escolares ocurridas en su desarrollo. Posteriormente, cada actividad (un total de 138) se describió de acuerdo con variables que permiten analizarlas como situaciones comunicativas. La comparación, uno a uno, de los rasgos que caracterizan las actividades de clase de los seis profesores y un posterior análisis multivariado de las descripciones hechas permitieron identificar cuatro perfiles de enseñanza: profesor científico, profesor escolar, profesor frontal y profesor circular. Los perfiles se describen y discuten desde la perspectiva de disyuntivas propias de la profesión docente.

Palabras clave: Enseñanza, comunicación, actividad escolar, perfiles de enseñanza

\section{Abstract}

The paper reports a research that was carried out in order to identify teaching profiles based on the analysis of different ways of assuming communicative interaction in the classroom. Six Science teachers from two public schools of Bogotá, Colombia, participated in the study. They teach Science to $6^{\text {th }}, 7^{\text {th }}$ and $8^{\text {th }}$ graders. A sequence of three classes was videotaped for each teacher. Each class was then analyzed to identify the school activities that composed it. Afterwards, each school activity (a total of 138) was described by means of a group of variables that consider the activity as a communicative event. The comparison of the main features that characterize the six teachers' class activities and an additional multivariate analysis of the descriptions led to the identification of four teaching profiles: the scientific teacher, the classroom teacher, the frontal teacher and the circular teacher. These profiles are described and discuss with respect to the teaching profession.

Keywords: Teaching, communication, classroom activity, teaching profile

\section{Resumo}

0 artigo relata um processo de pesquisa realizada com o objetivo de identificar perfis de aprendizagem a partir da análise das formas de interação comunicativa na sala de aula. Participaram do estudo seis professores de ciências naturais de duas escolas oficiais em Bogotá, Colômbia, que ensinam nas aulas de sexto a oitavo de educação básica. Sequências de três classes foram registradas para cada professor, cada uma das quais foi então analisada para identificar as atividades escolares que ocorrem em seu desenvolvimento. Posteriormente, cada atividade (um total de 138), foi descrita de acordo com variables que permitem analisálas como situações de comunicação. A comparação, um por um, dos traços que caracterizam as atividades dos seis professores de classe, e uma posterior análise multivariada das descrições feitas tornou possível identificar quatro perfis de ensino: professor de ciências, professor de escola, professor frontal e professor circular. Os perfis são descritos e discutidos a partir da perspectiva das disjuntivas próprias da profissão docente.

Palavras Chave: Ensino, comunicação, atividade escolar, perfis de ensino

1 Universidad Pedagógica Nacional, Bogotá, Colombia. Correo electrónico: acamargo@pedagogica.edu.co

2 Universidad Pedagógica Nacional, Bogotá, Colombia. Correo electrónico: hederich@pedagogica.edu.co 
Universidad Pedagógica Nacional

Facultad de Humanidades

\section{Introducción}

La clase escolar puede entenderse como un evento comunicativo en el cual los actores (profesor y estudiantes) asumen unos roles predeterminados y los desempeñan mediante una diversidad de formas de significación, con el fin de lograr metas de carácter pedagógico o didáctico. Puesto que el rol del profesor en esta situación de interacción conlleva funciones de autoridad, se espera que sea él quien tome las decisiones sobre qué rol asume cada cual en cada momento de la clase. Así, en la mayoría de los casos, es el profesor quien introduce el tema, propone y organiza las actividades de clase, conduce el intercambio comunicativo y asigna los turnos de participación a los estudiantes (Cazden, 1988; Christie, 2002; Gumperz y Hymes, 1972; Stubbs, 1983; entre otros).

En este contexto, es posible pensar que las decisiones que cada profesor toma sobre la manera de interactuar en su clase son indicadoras de una manera específica de enseñar y podrían entonces considerarse como parte componente de un perfil de enseñanza susceptible de indentificarse, describirse y analizarse. El presente trabajo reporta un ejercicio investigativo que explora precisamente esta posibilidad, a partir de la descripción de clases como eventos comunicativos representativos de una forma de gestionar la interacción durante la enseñanza (Camargo, 2015; McCroskey \& Richmond, 1995).

Con el objeto de proponer estos perfiles de interacción comunicativa en el aula, el presente artículo incluye un marco de referencia que ubica la investigación desde el punto de vista de sus principales antecedentes y presenta un modelo de análisis de las actividades de aula desde una perspectiva comunicativa. Dicho modelo permitirá presentar y definir las dimensiones, los criterios y las variables que guiarán la descripción y comparación de las formas de enseñar de seis profesores de ciencias naturales, durante el desarrollo de unidades temáticas específicas correspondientes al currículo de educación básica. El seguimiento detallado de estas comparaciones, junto con un análisis multivariado global final, permitirán proponer perfiles de interacción comunicativa que faciliten la descripción de diferentes propuestas de aproximación hacia la enseñanza.

\section{Elementos conceptuales}

\section{Perfiles comunicativos del profesor. Algunos antecedentes}

La identificacion de perfiles de comunicación en el aula tiene, como antecedentes más relevantes, los estudios sobre tipos o estilos de enseñanza que toman en consideración el factor comunicativo para la definición de formas particulares de asumir la labor educativa.

Cabe mencionar, en primer lugar, el trabajo de McCroskey y sus colaboradores, que plantea que un profesor puede caracterizarse según el grado de asertividad y el grado de receptividad de su interacción con los estudiantes (McCroskey \& Richmond, 1995: Richmond \& McCroskey, 1992; Thomas, Richmond \& McCroskey, 1994; Wheeless \& Drierks-Steward, 1981). Según los autores, un profesor asertivo es propositivo y positivo en su manera de llevar la clase, responde rápida y eficazmente a los requerimientos de las situaciones de aula y posee una orientación muy clara hacia el logro de los objetivos de la actividad que se esté realizando. Por su parte, un profesor receptivo es sensible a las necesidades de sus estudiantes, realiza esfuerzos por comprenderlos y busca interactuar con ellos con el ánimo de conocerlos mejor (Kearney, 1984; McCroskey, Richmond \& McCroskey, 2006; Thomas, Richmond \& McCroskey, 1994).

La segunda propuesta de identificación de formas de comunicar en el aula es la planteada por Nussbaum y Tusón, que consiste en describir la actividad de los profesores desde el punto de vista del género discursivo que privilegian en sus clases. Podrían reconocerse entonces profesores expositivos, profesores narrativos, profesores argumentativos, etc. Cada uno de estos géneros podría definirse por los roles propios del profesor y de los estudiantes, ciertos patrones en el comportamiento comunicativo durante la interacción y en el uso lingüístico asociado a la situación. Así, en la medida en 
Perfiles de enseñanza según formas de interacción comunicativa en el aula. Estudio de caso con seis profesores de ciencias naturales Ángela Camargo-Uribe, Christian Hederich-Martínez

que es posible identificar qué tipo de actividades de interacción discursiva admite más frecuentemente un profesor, se puede identificar su perfil de enseñanza sobre la base del género discursivo preferido (Nussbaum \& Tusón, 1996).

Finalmente, vale la pena mencionar los trabajos de Mortimer y Scott que proponen, para el caso de la enseñanza de las ciencias naturales, que el intercambio comunicativo de los profesores y sus estudiantes puede describirse desde dos dimensiones: la dimensión dialógico frente a autoritario, que distingue entre la tendencia del profesor a tomar en consideración los puntos de vista de sus estudiantes o solo admitir los propios; y la dimensión interactivo frente a no interactivo, que distingue entre un discurso que admite intervenciones de otros participantes, los estudiantes, y un discurso que no lo hace. De acuerdo con los autores, estos dos criterios descriptivos definen ciertos enfoques comunicativos característicos de cada profesor (Mortimer \& Scott, 2003).

Mirados en su conjunto, los perfiles de enseñanza que surgen de estas propuestas cubren un amplio espectro de factores de la actividad docente cuando se la observa con una mirada comunicativa. Cada una, sin embargo, apenas alcanza a dar cuenta de un aspecto aislado del proceso. Mientras el modelo de McCroskey enfatiza en aspectos actitudinales de la comunicación del profesor, el de Nussbaum y Tusón centra su atención en el discurso propiamente dicho y el de Mortimer y Scott en el tipo de interacción promovida por el profesor. El enfoque que se asume en este estudio busca una mirada un tanto más comprensiva de la enseñanza, al asumir la situación de clase como un evento comunicativo. Para ello tomaremos como base la propuesta teórica del lingüista británico M. K. Halliday. Este es el tema del siguiente apartado.

\section{La actividad de clase como evento interactivo-discursivo}

De acuerdo con Halliday (1982), los episodios que componen cualquier evento comunicativo, por ejemplo, una sesión de clase, se entienden como secuencias de situaciones de comunicación o de construcción de sentidos y cada una de ellas puede describirse desde tres puntos de vista:

1. Como muestra de un campo de actividad social. La situación social se reconoce como tal por la comunidad participante y los mensajes que se intercambian se interpretan desde este reconocimiento. De esta manera, todo lo que dice un profesor en su clase se interpreta en relación con la actividad de "enseñar", y no con "diagnosticar" o "conversar con amigos", por ejemplo.

Como parte del campo de una determinada situación social, es de especial importancia que se defina tanto el tema de que trata, como el propósito general de la misma. Las actividades de aprendizaje que componen una sesión de clase pueden considerarse entonces como situaciones o eventos sociales porque tratan de algo (el objeto de aprendizaje) y tienen alguna finalidad en ese contexto (el aprendizaje mismo en alguna de sus fases).

2. Como muestra de un tenor para las funciones y las relaciones entre los participantes. Los roles de profesor y estudiante, para el caso que nos ocupa, se encuentran tan bien configurados en los entornos socioculturales occidentales modernos, que puede incluso bromearse con estereotipos sobre ellos.

El tipo de relaciones que puedan establecerse entre los participantes en la situación comunicativa se encuentran ligadas tanto a atributos particulares de los participantes como a condiciones surgidas de la experiencia de interacción vivida entre ellos. Ello configura lo que podrían denominarse climas de relaciones comunicativas atendiendo a la verticalidad u horizontalidad de la relación, la lejanía o cercanía de las mismas, la carga emocional que cada participante aporta a la situación, etc.

3. Como muestra de un modo de manejo discursivo. Esta perspectiva alude a las características del discurso que se produce durante 
Universidad Pedagógica Nacional

Facultad de Humanidades

la situación. Estas características tienen dos dimensiones. La primera incumbe a la forma como tal del discurso que codifica tanto el campo como el tenor del evento que se describe, y la segunda a las peculiaridades del discurso en sí mismo: los medios de transmisión elegidos, el esquema textual utilizado, las formas gramaticales preferidas, etc.

Para la descripción del modo de las situaciones de aula como situaciones comunicativas habría que hacer énfasis en aquellos procesos lexicogramaticales que convierten el contenido y el propósito del aprendizaje en una secuencia verbal específica, y en aquellos del discurso que plasman lingüísticamente los elementos de la estructura de roles establecida.

Siguiendo el modelo de Halliday, todo encuentro comunicativo estaría estructurado por un campo de actividad conjunta, un tenor de roles y climas de relación entre los participantes y un modo de realización simbólica (verbal-discursiva) de los elementos anteriores ${ }^{3}$.

El esquema anterior sería válido tanto para una sesión completa de clase como para las actividades que la componen, en la medida en que en cada una de ellas puedan identificarse un campo, un tenor y un modo. Así, por ejemplo, si en una clase un profesor pasa de la actividad inicial de corrección de la tarea para ese día, a la actividad de presentación de un contenido nuevo de aprendizaje mediante una explicación en el tablero, estaríamos hablando de dos actividades diferentes, cada una con un campo, un tenor y un modo potencialmente diferentes.

De esta manera, siguiendo a Halliday (1982), el evento comunicativo "sesión de clase" se entenderá como un contexto de interacción comunicativa en donde pueden identificarse pequeños episodios

3 Debe advertirse que la dimensión de modo no será considerada para este estudio específicamente. Puesto que el análisis de la actividad discursiva del profesor es sin duda una tarea altamente compleja e involucra diversas dimensiones difíciles de abarcar de manera global, hemos dejado esta dimensión de modo como un estudio aparte. Algunos resultados parciales de esta perspectiva discursiva pueden leerse en un trabajo publicado en 2014 por Camargo (véanse las referencias). comunicativos, que llamaremos actividades de clase, reconocibles como tales porque cumplen los siguientes requisitos:

- Poseen un contenido temático identificable fácilmente.

- Pueden asimilarse a una actividad (de aprendizaje), con un propósito detectable.

- Configuran roles o funciones de interacción comunicativa de la que cada participante es consciente y asume sin ambigüedades.

Se asumirá entonces la actividad de clase como una unidad comparable a una situación comunicativa y, por ello, analizable de acuerdo con el modelo de Halliday. En consecuencia, una actividad de clase se concibe aquí como una situación de interacción comunicativa, que puede identificarse por su contenido y su propósito y en donde es posible definir el rol de participación de cada uno de los interlocutores. La presentación de un tema nuevo por parte del profesor, la resolución conjunta de un ejercicio o un problema, la revisión y corrección de una tarea, el repaso de lo visto en sesiones de clase anteriores, el llamado a lista para registrar asistencia o la discusión alrededor de una problemática planteada, son todas actividades de clase, puesto que cumplen los requisitos arriba definidos.

\section{Metodología}

\section{Participantes}

La fuente de información con la que se cuenta en esta investigación proviene de la actividad profesional de seis profesores de ciencias naturales en la educación básica secundaria: cuatro profesoras y dos profesores, de diferentes rangos de edad, todos con más de diez años de experiencia como docentes. Los profesores enseñaban en dos colegios. Uno enseñaba en sexto grado, cuatro enseñaban en séptimo grado y el último en octavo. A cada profesor le fue observada una secuencia de tres clases, en las cuales se daba cuenta de una unidad didáctica completa sobre un tema específico. Los nombres de los profesores han sido cambiados por seudónimos extraídos de la 
Perfiles de enseñanza según formas de interacción comunicativa en el aula. Estudio de caso con seis profesores de ciencias naturales Ángela Camargo-Uribe, Christian Hederich-Martínez

mitología griega, pues sus identidades reales no interesan en este estudio; de igual forma, los nombres de las instituciones educativas fueron sustituidos por nombres de ciudades de la Grecia Antigua. Los datos de los profesores y sus temas aparecen en la tabla 1.

Tabla 1. Los seis profesores participantes en el estudio

\begin{tabular}{l|l|l|l|l|l}
\multicolumn{1}{c}{ PROFESOR } & \multicolumn{1}{c}{ GÉNERO } & \multicolumn{1}{c}{ EDAD } & \multicolumn{1}{c}{ GRADO } & COLEGIO & \multicolumn{1}{c}{ TEMA } \\
\hline Artemisa & Femenino & Entre 30 y 40 & Séptimo & Tebas & La biodiversidad \\
\hline Atenea & Femenino & Entre 50 y 60 & Séptimo & Tebas & Estructura atómica y molecular de la materia \\
\hline Dionisio & Masculino & Entre 40 y 50 & Sexto & Tebas & Propiedades y estados de la materia \\
\hline Gaya & Femenino & Entre 40 y 50 & Octavo & Creta & Ecosistemas acuáticos \\
\hline Hera & Femenino & Entre 50 y 60 & Séptimo & Creta & Enlaces en compuestos químicos \\
Hermes & Masculino & Entre 30 y 40 & Séptimo & Tebas & Los elementos químicos y la tabla periódica \\
\hline
\end{tabular}

Cada secuencia de tres clases de los seis profesores participantes en el estudio fue videograbada. Sobre la base de estas grabaciones se elaboraron sinopsis de cada clase. Para la elaboración de estas sinopsis, cada video fue examinado para identificar las actividades que componen la sesión de clase, siguiendo los criterios arriba mencionados, esto es: (1) que posean un contenido temático reconocible fácilmente, (2) que puedan asimilarse a una actividad de aprendizaje con un propósito identificable y (3) que configuren roles o funciones de interacción comunicativa que cada participante reconozca y asuma.

Así, se conformó una base de datos con todas las actividades que componen las clases observadas (18), para un total de 138 actividades de clase. Cada actividad, señalada como tal en la base de datos, puede identificarse por su pertenencia a alguna de las tres clases dictadas por alguno de los seis profesores cuyas clases estamos analizando. En la tabla 2 se muestra el recuento de las actividades por cada profesor en cada una de las clases.

Tabla 2. Recuento de actividades de clase por profesor

\begin{tabular}{|l|c|c|c|c|}
\hline \multirow{2}{*}{ PROFESOR } & \multicolumn{3}{|c|}{ CLASE } & Total \\
\cline { 2 - 5 } & Primera & Segunda & Tercera & \\
\hline Artemisa & 5 & 6 & 6 & 17 \\
\hline Atenea & 7 & 11 & 6 & 24 \\
\hline Dionisio & 8 & 7 & 5 & 20 \\
\hline Gaya & 9 & 7 & 10 & 26 \\
\hline Hera & 10 & 11 & 11 & 32 \\
\hline Hermes & 8 & 6 & 5 & 19 \\
\hline Total & 47 & 48 & 43 & $\mathbf{1 3 8}$ \\
\hline
\end{tabular}
posible de once actividades por clase y un mínimo de cinco. Las diferencias observadas en el número total de actividades por profesor se relacionan con diferencias en las longitudes de las sesiones de clase de las dos instituciones educativas en donde se realizaron las observaciones. Mientras en el colegio que hemos denominado "Tebas" las sesiones de clase duran un máximo de 60 minutos, las sesiones de clase del colegio "Creta" duran hasta 120 minutos por clase. Para salvar estas diferencias, los análisis descriptivos y comparativos se harán sobre la base de porcentajes de frecuencias por profesor y no sobre recuentos netos de actividades de clase.

Tal y como lo explicamos, puesto que una actividad de clase se corresponde directamente con un evento comunicativo, ella se puede describir desde el punto de vista de su campo, su tenor y su modo (Halliday, 1982). Recordemos que el campo de una actividad de clase alude a los contenidos y propósitos comunicativos que la identifican y la diferencian de otras actividades posibles dentro de la misma sesión de clase. Por su parte, el tenor de una actividad de clase tiene que ver con los rasgos que definen los roles y las funciones que, tanto el profesor como los estudiantes, pueden asumir para el logro del propósito establecido. Finalmente, el modo de una actividad de clase tiene que ver con las diversas formas como los elementos que caracterizan una cierta interacción específica se manifiestan en el discurso de los participantes. 
Universidad Pedagógica Nacional

Facultad de Humanidades

Para el presente contexto, las 138 actividades identificadas se describirán respecto de su campo y de su tenor. Puesto que tales caracterizaciones tienen un fin comparativo, el sesgo descriptivo será la búsqueda de diferencias en la forma como cada uno de los profesores participantes en el estudio configura el campo y el tenor de las actividades en sus clases.

\section{Descripción y comparación de las actividades de clase. Dimensiones, variables e indicadores}

Desde el punto de vista de su campo, las actividades de clase que describiremos se analizarán de acuerdo con dos variables que lo definen comunicativamente: su propósito y su grado de acercamiento a la actividad científica.

Respecto de la primera variable, el propósito, es relativamente estrecho el margen de decisión individual del profesor frente a los propósitos de interacción durante sus clases. Asumimos que siempre habrá un propósito pedagógico en toda actividad de aula. Exploraremos este pequeño margen con base en tres posibles preferencias de los profesores, que actúan como indicadoras de propósito: (a) la notoriedad que les otorgan a actividades que no se relacionan directamente con el contenido científico de su clase, (b) la importancia que le conceden a la evaluación del aprendizaje como objetivo de actividades de clase y (c) la relevancia que le asignan a la regulación del comportamiento en clase, como meta de las actividades de clase.

Por su parte, con respecto al segundo de los criterios de descripción, o la segunda variable mencionada para la descripción del campo, su grado de acercamiento a la actividad científica, la exploración comparativa pondrá énfasis en el carácter científico del tema de la actividad, o en el carácter escolar de la misma. Específicamente, intentaremos identificar opciones respecto de los siguientes dos indicadores: (a) el formato científico o escolar, en el que las actividades se diseñan y llevan a cabo y (b) el nivel de "densidad temática" de las sesiones de clase de cada profesor, indicada por la novedad o recurrencia de un cierto tema durante las actividades de clase de cada profesor.

Ahora bien, desde el punto de vista del tenor de las actividades, es decir, del esquema de roles y el clima interactivo general que se configuran en ellas, se tomaron en consideración las siguientes variables e indicadores:

Respecto de los roles, el análisis de las clases focalizará su atención en la identificación de los matices que asumen los roles generales de "profesor" y "estudiante", como consecuencia del tipo de interacciones que cada uno de los seis profesores diseña y lleva a cabo en sus clases. Para explorar esta idea, describiremos las actividades de clase utilizando dos indicadores: (a) la organización espacial del profesor y sus estudiantes en el desarrollo de la actividad y (b) el tipo de intercambio participativo que se configura en cada actividad. Mientras la primera variable proporciona indicios sobre niveles de horizontalidad o verticalidad en las interacciones, la segunda permite descubrir señales del rol (activo o pasivo) que el profesor otorga a sus estudiantes.

Asimismo, respecto del clima de interacción global que se observa en cada actividad, las descripciones intentarán determinar el grado de espontaneidad que cada profesor permite en sus clases, respecto de la participación del estudiante en ellas. Para lograrlo, caracterizaremos cada actividad en tres indicadores: (a) la frecuencia de uso del esquema de intercambio $\mathrm{IRE}^{4}$, (b) la frecuencia de participación espontánea del estudiante durante la actividad y (c) la frecuencia de ocurrencia de actividades simultáneas en la clase.

En general, pensamos que, en cumplimiento de su rol profesional, cada profesor ideó los encuentros de clase, imaginando y organizando actividades que, al tiempo que respondían a propósitos pedagógicos y didácticos específicos,

4 La sigla IRE se refiere al esquema de intercambio: interrogaciónrespuesta-evaluación, que ha sido definido como el formato de interacción profesor-estudiante más típico del aula de clase (Sinclair y Coulthard, 1975). Incluir este factor como parte del clima de la actividad es relevante puesto que su uso refleja un cierto grado de conservatismo en las formas y maneras tradicionales de un aula de clase. 
se acomodaban a las características de la relación que ha ido construyendo con sus estudiantes y a su propia forma de ser como sujeto que comunica. Es en esa descripción del campo y el tenor que cada profesor privilegió las actividades que eligió,
Perfiles de enseñanza según formas de interacción comunicativa en el aula. Estudio de caso con seis profesores de ciencias naturales Ángela Camargo-Uribe, Christian Hederich-Martínez

sobre las cuales será posible proponer perfiles comunicativos de enseñanza.

En la tabla 3 se resumen los parámetros de descripción y comparación de las actividades de clase desde el punto de vista de su campo y tenor.

Tabla 3. Parámetros de descripción y comparación de las actividades de clase

\begin{tabular}{|c|c|c|c|}
\hline DIMENSIÓN & VARIABLES & INDICADORES & VALORES \\
\hline \multirow{5}{*}{ Campo } & \multirow{3}{*}{ Propósito } & Propósito general & $\begin{array}{l}\text { Tema de clase } \\
\text { Otro tema }\end{array}$ \\
\hline & & Énfasis en evaluación & $\begin{array}{l}\text { Actividad en donde se evalúan aprendizajes previos } \\
\text { Actividad sin evaluación }\end{array}$ \\
\hline & & Énfasis en regulación & $\begin{array}{l}\text { Control: Actividad con propósito regulador del comportamiento } \\
\text { No control: Actividad sin propósito de regulación }\end{array}$ \\
\hline & \multirow{2}{*}{ Contenidos } & Aproximación a la ciencia & $\begin{array}{l}\text { Actividad científica profesional } \\
\text { Actividad científica escolar sigue el estándar escolar } \\
\text { Actividad de temas no científicos }\end{array}$ \\
\hline & & Densidad de contenidos & $\begin{array}{l}\text { Actividad que introduce un tema nuevo } \\
\text { Actividad que trabaja un tema ya conocido }\end{array}$ \\
\hline \multirow{5}{*}{ Tenor } & \multirow[b]{2}{*}{ Roles } & Organización espacial & $\begin{array}{l}\text { Actividad frontal } \\
\text { Actividad circular }\end{array}$ \\
\hline & & Tipos de intercambio & $\begin{array}{l}\text { Profesor-grupo } \\
\text { Profesor-estudiantes } \\
\text { Estudiante-estudiante }\end{array}$ \\
\hline & \multirow{3}{*}{ Climas } & Uso del esquema IRE & $\begin{array}{l}\text { Presencia de IRE } \\
\text { Ausencia de IRE }\end{array}$ \\
\hline & & $\begin{array}{l}\text { Participación estudiantil } \\
\text { espontánea }\end{array}$ & $\begin{array}{l}\text { Voz del estudiante } \\
\text { Ausencia de la voz del estudiante }\end{array}$ \\
\hline & & Organización temporal & $\begin{array}{l}\text { Secuencial (una a la vez) } \\
\text { Simultánea (varias a la vez) }\end{array}$ \\
\hline
\end{tabular}

\section{Resultados}

Las actividades de las secuencias de clase a cargo de los seis profesores participantes en el estudio se describieron respecto de las variables incluidas en la tabla 3. Tales descripciones fueron la base para realizar la comparación, desde el punto de vista de estas variables, de las maneras como estos profesores gestionan comunicativamente sus clases. Como se verá, las comparaciones permitirán ir construyendo perfiles más o menos claros de interacción comunicativa en el ejercicio de la docencia.

\section{Diferencias en el propósito de las actividades de clase}

En la gráfica 1 se muestran los porcentajes de actividades por profesor según el contenido de la interacción en cada una de ellas. Como es de esperarse, la mayoría (73\%) de las actividades de clase registradas aluden al tema científico de la unidad que cada profesor está desarrollando. Ello deja una minoría de actividades con contenidos diferentes. Esta minoría permite identificar diferencias entre los profesores, según si dedican espacio significativo de la clase a interacciones comunicativas con propósitos no didácticos, o si lo hacen en menor medida. Respecto de las actividades de clase dedicadas a 
Universidad Pedagógica Nacional

Facultad de Humanidades

temas que no se relacionan directamente con las ciencias naturales, los seis profesores se dividen en tres grupos. El primero, conformado por Hermes y Hera, en quienes se observa el $37 \%$ y el $41 \%$ de las actividades dedicadas a temas no científicos. El segundo, conformado por Atenea y Artemisa, en cuyas clases estos otros temas corresponden al 25\% y al 29\% de las actividades de la clase, respectivamente. Y, finalmente, Gaya, con el 15\% y Dionisio, con el $10 \%$, son el grupo que menos actividades dedica a propósitos no didácticos en sus clases.

En la gráfica 2 se presentan los porcentajes de actividades por profesor según la presencia de evaluación en ellas. En general, el 26\% de las actividades identificadas pueden clasificarse como de tipo evaluativo. Este porcentaje moderado de actividades evaluativas se distribuye de manera muy dispareja entre los profesores participantes en el estudio. Con un $65 \%$ de actividades de naturaleza evaluativa se encuentra Dionisio. A una gran distancia se encuentran Artemisa, con el 29\%, Gaya, con el 27\% y Atenea, con el 25\%, de actividades con función evaluativa. Por último están Hermes, con el 11\% y Hera, con el 9\% de actividades evaluativas.

En la gráfica 3 se observan los porcentajes de actividades por profesor que incluyen procesos regulatorios (de control del comportamiento) y porcentajes de actividades que no los incluyen. En general, solo un $23 \%$ de las actividades identificadas incluye un componente regulatorio claramente identificado. Más que suponer que en el otro 77\% de las actividades no ocurre actividad regulatoria alguna, podría suponerse que este propósito comunicativo se encuentra mezclado con propósitos más claramente didácticos. En otras palabras, estaríamos frente a un $23 \%$ de actividades en donde se observa una intención del profesor asociada directa y exclusivamente con propósitos de regulación del comportamiento de sus estudiantes. Con esta reflexión en mente, y en términos comparativos, los seis profesores del estudio podrían dividirse en dos grupos. Uno en el cual los propósitos de regulación del profesor adquieren más frecuentemente una expresión explícita. A este grupo pertenecen Atenea, con el 38\%, Artemisa, con el 29\% y Hermes, con el $26 \%$. En contraste, el segundo grupo de profesores podría caracterizarse como aquel en el cual los propósitos de regulación podrían estar mezclados con objetivos de tipo didáctico y, en esa medida, sus comportamientos exclusivamente regulatorios son menos frecuentes. A este grupo pertenecen Gaya, con el 19\%, Hera, con el 16\% y Dionisio, con el 19\% de actividades de tipo regulatorio.

La consideración conjunta de los tres aspectos relacionados con los propósitos de las actividades de clase (inclusión de propósitos no didácticos, inclusión de actividades evaluativas y uso de estrategias explícitas de regulación) permite configurar un primer perfil de enseñanza, personificado en Dionisio, claramente identificable. Se trata de una forma de enseñanza centrada en el contenido de la asignatura, que programa las actividades de clase con esta mirada y utiliza una gran parte de la interacción con sus estudiantes para propósitos evaluativos. Con menos fuerza que el perfil anterior, se configura también una forma de enseñar, personificada en Hermes y Hera, en donde el tema específico de la clase tiene menos relevancia y, en consonancia, parece menor la actividad evaluativa en la clase.

\section{Diferencias en el contenido científico de las actividades}

Las actividades de clase desarrolladas por los seis profesores fueron comparadas respecto de la frecuencia con que ellas se acercaban a la actividad del científico (experimentos, observaciones, análisis, etc.). La comparación se hizo solo para aquellas actividades identificadas como propiamente didácticas, es decir, cuya temática corresponde al contenido de las ciencias naturales (un total de 104 actividades). En la gráfica 4 se presenta esta comparación.

En general, el 38\% de las actividades sobre el tema de la clase poseen un formato científico y el $63 \%$ de ellas posee un formato escolar (ejercicios en clase, elaboración de resúmenes, dictados, etc.). Ello indica que la transposición de los contenidos científicos a formatos didácticos es una estrategia generalizada para el nivel de enseñanza en el 
Perfiles de enseñanza según formas de interacción comunicativa en el aula. Estudio de caso con seis profesores de ciencias naturales Ángela Camargo-Uribe, Christian Hederich-Martínez

que estamos trabajando. Desde el punto de vista comparativo, las diferencias entre los profesores respecto del uso del formato científico saltan a la vista. En un primer grupo de profesores con un uso relativamente frecuente del formato científico en sus actividades de clase, se encuentran Hera, con el 53\%, y Hermes, con el 50\%, de actividades con formato científico. En niveles intermedios de uso de este formato se hallan Atenea, con el 42\%, y Gaya, con el 41\%. Finalmente, en el último grupo (a pesar de la gran distancia entre ellos) podrían ubicarse Dionisio, con el 28\% de actividades con formato científico y Artemisa, en cuyas clases no se observaron actividades con tal formato.

La consideración de esta nueva variable apoya el primero de los perfiles configurados, el de Dionisio, puesto que asigna, para él, el rasgo de uso preferencial del formato escolar en las interacciones comunicativas de sus clases. Ello, combinado con las anteriores características de focalización en el tema de la clase y uso frecuente de actividades evaluativas, configura por el momento una forma de enseñar, descrita aquí en términos de la preferencia por actividades de clase que algunos autores han llamado ciencia escolar (Mortimer \& Scott, 2003).

Ahora bien, otro criterio para identificar diferencias entre los profesores respecto de qué tanto emulan ellos la actividad científica en el aula es la densidad de los contenidos científicos planteados en clase. La idea que dirige esta descripción es que, en contraste con una actividad propiamente escolar, en donde es normal el tratamiento repetitivo de un tema desde muy diversos puntos de vista, la actividad científica es por lo general densa y amplia, temáticamente hablando (Suppe, 1998). En la gráfica 5 se muestran las diferencias entre los profesores respecto de este criterio.

Los porcentajes de frecuencias generales presentan un gran parecido con los observados para la variable anterior. Un 33\% de las actividades introducen un tema nuevo a la clase, mientras que un $67 \%$ de las actividades tocan temas ya vistos.

La consideración de este criterio de densidad temática, desde el punto de vista comparativo, marca diferencias importantes entre los profesores participantes en el estudio. En primer lugar, con el $64 \%$ de actividades con tema nuevo, están las clases de Hermes. Sus clases están muy densamente administradas desde el punto de vista temático. En segundo lugar pueden agruparse Atenea, con el 39\%, Hera, con el 32\% y Gaya, con el 30\%, de actividades sobre temas nuevos. Finalmente, en el último puesto respecto de la densidad temática de sus clases, se encuentran Dionisio y Artemisa, ambos con el 17\% de actividades en donde se introduce un tema nuevo.

Esta variable agrupa a profesores participantes en el estudio en dos sentidos. Por un lado, en la construcción de un perfil escolar de profesor de ciencias, en el que se ubica claramente Dionisio, y posiblemente Artemisa, al ser una profesora que privilegia tanto las actividades de clase con formato escolar como el tratamiento repetitivo de un tema desde muchos puntos de vista. Por otra parte, respecto de los indicios que identifican a Hermes y Hera como profesores con preferencia por actividades de clase cercanas al quehacer científico, el perfil de Hera en este grupo se desvanece un poco y la imagen de Hermes toma fuerza, al tener él la mayor tendencia a introducir muchos temas nuevos en sus clases.

\section{Diferencias en los roles que las} situaciones comunicativas construyen para las actividades propuestas

A fin de identificar preferencias en los grados de horizontalidad o verticalidad entre los roles de profesor y estudiante, las actividades fueron clasificadas de acuerdo con la ubicación espacial de los participantes durante su realización en el aula de clase. Este criterio identificó dos posibilidades: la organización frontal clásica, que conlleva una jerarquía clara entre los roles, y la organización circular con el profesor, que forma parte del círculo e indica menores niveles de verticalidad entre los roles. En gráfica 6 se presenta esta comparación.

En general, es posible observar mayor frecuencia de la organización frontal de los participantes. Un $80 \%$ de las actividades de clase observadas poseen esta organización espacial. En términos 
Universidad Pedagógica Nacional

Facultad de Humanidades

comparativos, Dionisio (95\%), Atenea (92\%) y Gaya (85\%) superan la tendencia media de organización frontal y, en contraste, Artemisa (76\%), Hera (75\%) y Hermes (68\%) están por debajo de la media.

Aunque parezca inocuo, la organización espacial frontal refleja roles de participación durante la actividad. En este tipo de organización, resulta clara la estructura de autoridad en el aula, la cual determina el uso de la palabra, la toma de decisiones $y$, en últimas, el tipo y tono de las intervenciones de cada cual. Ello se encuentra en consonancia con otro de los indicadores de lo que se conoce como frontalidad o enseñanza frontal en el aula: la frecuencia de las intercambio profesor-grupo.

Cada una de las actividades de clase se describió desde el punto de vista del tipo de intercambio verbal presente en ella. En general, se pudieron observar tres tipos de intercambios: (1) el intercambio profesor-grupo, en el cual el profesor se dirige al grupo y cuando un estudiante participa lo hace, en algún sentido, en nombre del grupo, (2) el intercambio personal profesor-estudiante, en el cual un profesor se dirige a un estudiante para referirse a asuntos que le conciernen solo a él, y el estudiante le responde en ese entendido y (3) el intercambio estudianteestudiante, en el cual el profesor solo actúa como observador del proceso. En la gráfica 7 se presentan los porcentajes de actividades por profesor según el tipo de intercambio presente el ellas.

Como se observa, el tipo de intercambio favorecido por los profesores en su conjunto es el de profesor-grupo (77\%), le sigue el intercambio profesor-estudiante (13\%), y el menos favorecido de los tres es el intercambio estudiante-estudiante. De acuerdo con lo esperado, los tres profesores que privilegian una organización espacial frontal (Atenea, Dionisio y Gaya) muestran igualmente las más altas frecuencias de interacción profesorgrupo. Hermes y Hera, por su parte, al tiempo que muestran una alta frecuencia de organización espacial circular, muestran los más altos porcentajes de interacción estudiante-estudiante. Comienza a configurarse entonces un perfil de profesor frontal, en oposición a uno de profesor circular, sobre la base de preferencias, tanto de la disposición espacial de los participantes, como del tipo de intercambio privilegiado.

\section{Diferencias en el clima interactivo gestionado por los profesores para las actividades que proponen}

Tres variables específicas permitirán explorar las formas como los profesores construyen el clima de interacción comunicativa en sus clases: la frecuencia del esquema IRE en las actividades, la presencia de intervenciones espontáneas de los estudiantes en la actividad y la propuesta de actividades simultáneas durante la clase.

En la gráfica 8 se muestran los porcentajes de presencia del esquema discursivo IRE en clase. En general, esta forma de interacción discursiva alcanza casi la tercera parte de las actividades de las clases observadas (28\%). Se destaca en la gráfica el caso de Hermes, quien no utiliza este patrón discursivo en sus clases. Le siguen Hera (13\%) y Artemisa (24\%) con porcentajes modestos; Atenea (33\%) y Gaya (35\%) con porcentajes importantes y, finalmente, Dionisio, con el $70 \%$ de sus actividades ocurridas bajo el esquema IRE.

Este resultado, unido a los anteriores, permite proponer un perfil muy claro de profesor frontal que privilegia actividades comunicativas en las que es él, el profesor, quien ejerce su rol de autoridad mediante el control de la participación verbal en el aula. Este perfil se encuentra representado, en la muestra de profesores participantes, por Dionisio, y en menor medida, por Atenea y Gaya. En contraste con este perfil, podría postularse el del profesor circular, cuyo rol de autoridad, si bien seguramente no desaparece del todo, podría verse disminuido mediante actividades y actitudes menos verticales.

En contraste con un esquema de diálogo estereotipado con funciones exclusivamente evaluativas, como lo es el esquema IRE, hay ocasiones en las que los estudiantes intervienen de manera espontánea y natural, para hacer comentarios propios sobre el tema que se está discutiendo, para preguntar algo derivado de las explicaciones del profesor o para 
Perfiles de enseñanza según formas de interacción comunicativa en el aula. Estudio de caso con seis profesores de ciencias naturales Ángela Camargo-Uribe, Christian Hederich-Martínez

aportar a la discusión que el profesor lidera. En la gráfica 9 se muestran los porcentajes de actividades en las que la participación espontánea del estudiante estuvo presente. En general, son pocas las oportunidades de clase para la intervención espontánea del rol estudiante. Un porcentaje promedio del 14\% da apoyo a esta afirmación. De acuerdo con los datos, los seis profesores se podrían dividir en tres grupos. Con porcentajes mínimos de actividades en donde se observa la participación espontánea del estudiante, están Dionisio y Hera ( $0 \%$ y 3\%, respectivamente). En seguida, se encuentran Hermes (16\%), Artemisa (18\%) y Atenea (21\%), con una disposición moderada hacia la intervención espontánea del estudiante. Por último, se encuentra Gaya con un $27 \%$ de actividades de clase en donde se deja abierta la posibilidad de intervención del estudiante, un porcentaje bastante alto respecto de la tendencia del resto de profesores.

Con estos datos, el perfil frontal de Dionisio se mantiene, mientras que el de Gaya se desdibuja al ser ella la que más participación estudiantil espontánea admite. En el otro extremo, el perfil circular de Hera se pierde, mientras que el de Hermes, si bien no se refuerza, tampoco pierde vigencia.

Por último, en la caracterización del tenor de las actividades de clase, se describió cada una de ellas desde el punto de vista de si suponía el trabajo al unísono de todos los participantes en ella o si admitía la posibilidad de que varios grupos de participantes estuvieran realizando tareas diversas al mismo tiempo. En la gráfica 10 se presentan los porcentajes de actividades por profesor según si en ellas se admiten diversas tareas realizadas al mismo tiempo por diferentes participantes o si se proponía una sola actividad que debían realizar de manera simultánea todos los estudiantes .

Los resultados generales muestran una preferencia de los seis profesores por organizar actividades en las que todos los estudiantes estén trabajando en lo mismo. Actividades como el trabajo por grupos o el trabajo personalizado solo cubren el 33\% de las actividades consideradas. El $67 \%$ restante corres- ponde a actividades en las que todos los estudiantes se encuentran atendiendo a un mismo foco temático o problemático. Se observan, eso sí, diferencias entre los participantes respecto de su mayor o menor preferencia por la simultaneidad de acciones entre los estudiantes. Al respecto, es posible agrupar a Artemisa, con un 94\%, Dionisio, con un $80 \%$, y Gaya, con un $73 \%$, como los profesores que prefieren secuenciar sus actividades y operar con todo el grupo de estudiantes en simultánea. En contraste, Atenea, con un 58\% y Hermes y Hera, ambos con el $53 \%$, presentan clases menos secuenciales.

En un sentido similar a la descripción de la variable referida a la organización espacial de los participantes en el aula de clase, una preferencia por mantener la actividad de los estudiantes al unísono refleja una cierto tenor de las actividades en el que se privilegia el rol del profesor como supervisor de las tareas de los estudiantes, por sobre un posible rol de facilitador del proceso de aprendizaje que ofrezca oportunidades de trabajo más personalizadas. Así, mientras que el perfil de Dionisio como profesor frontal se mantiene, y el de Hermes lo hace como profesor circular, las profesoras de la muestra presentan menos consistencia frente a sus niveles de frontalidad o circularidad. Como veremos en el análisis global que consignamos a continuación, la oposición logra mantenerse como expresión de diferencias claras entre los seis profesores.

Gráfica 1. Porcentaje de actividades por profesor según campo: propósito general

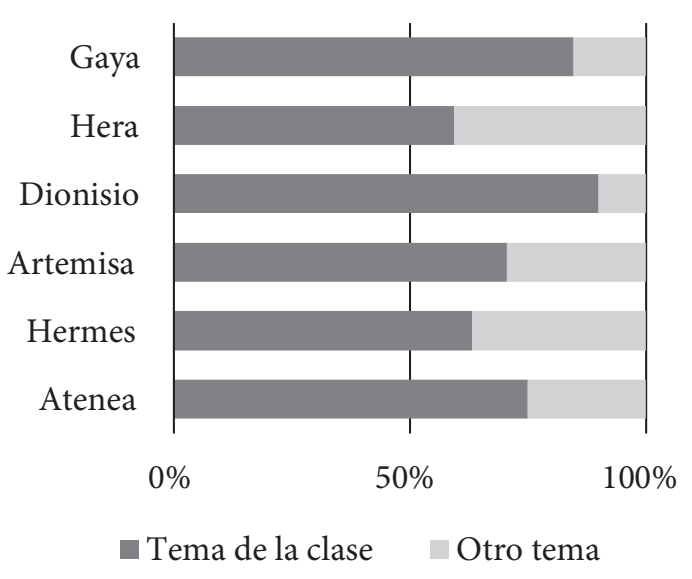


Gráfica 2. Porcentajes de actividades por profesor según campo: énfasis en evaluación

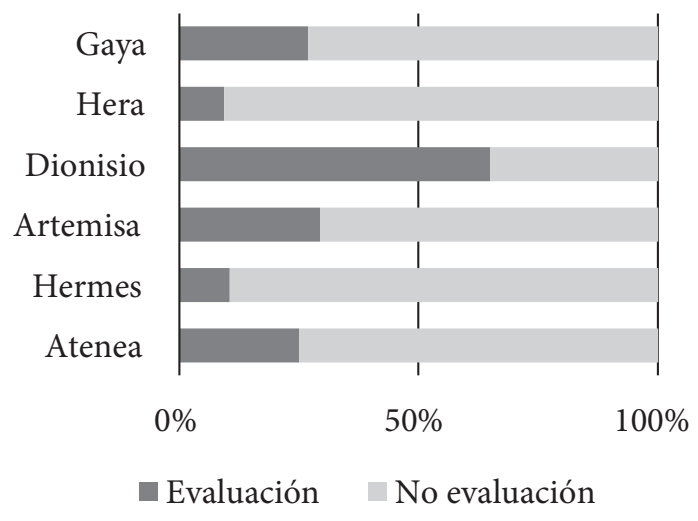

Gráfica 3. Porcentajes de actividades por profesor según campo-énfasis en regulación

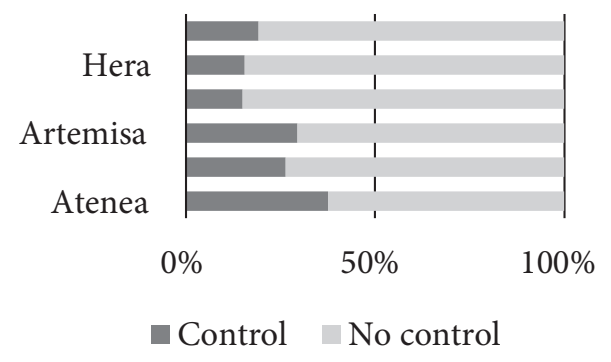

Gráfica 4. Porcentajes de actividades profesor según campo: aproximación a la ciencia

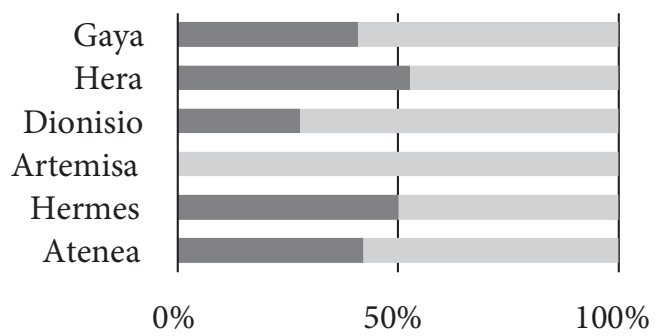

- Formato científico $\square$ Formato escolar

Gráfica 5. Porcentajes de actividades por profesor según campo-densidad de contenidos

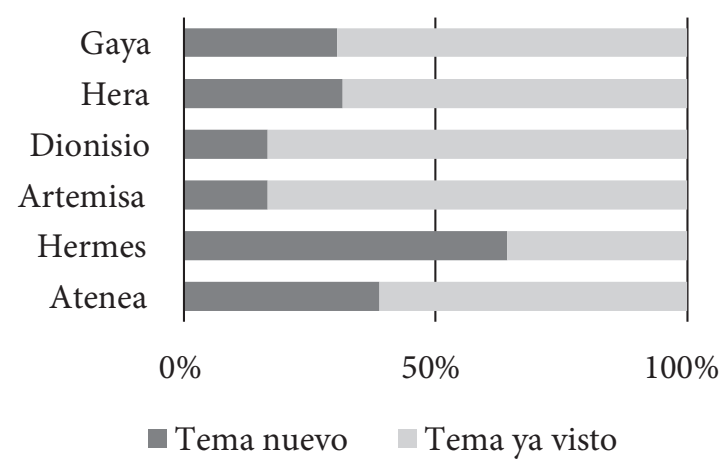

Gráfica 6. Porcentajes de actividades por profesor según tenor: organización espacial

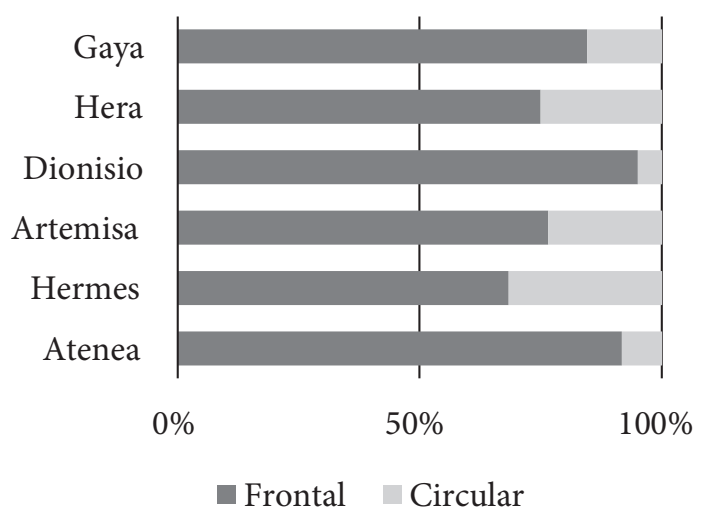

Gráfica 7. Porcentajes de actividades por profesor según tenor-tipo de intercambio

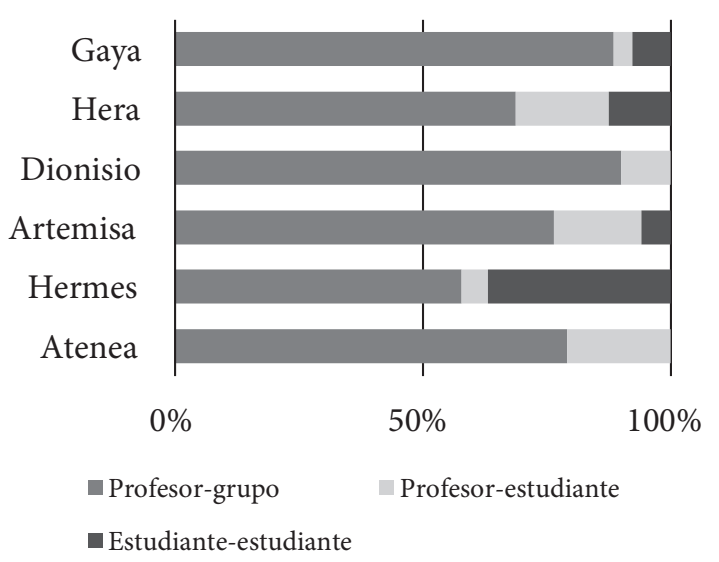

Gráfica 8. Porcentajes de actividades por profesor según tenor-uso del esquema IRE

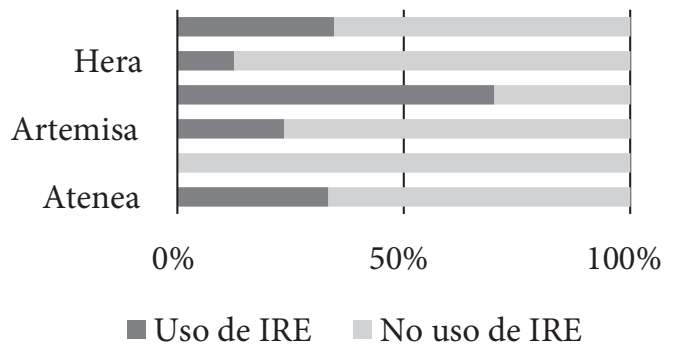

Gráfica 9. Porcentajes de actividades por profesor según tenor: participación estudiantil espontánea

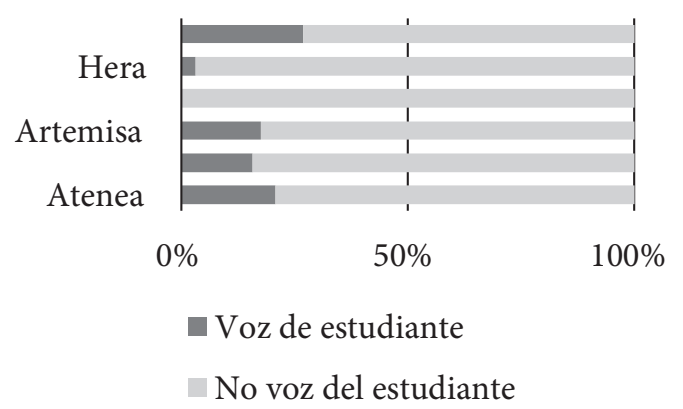


Gráfica 10. Porcentajes de actividades por profesor según tenor- organización temporal

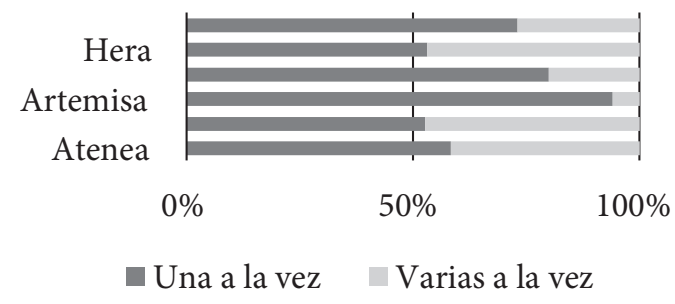

\section{Análisis descriptivo multivariante de las actividades}

Con la intención adicional de poner a prueba, mediante otra vía, las hipótesis construidas hasta el momento, la información respecto de las actividades de clase se sometió a un análisis de correspondencias múltiples ${ }^{5}$.

Para este análisis, actuaron como casos, o individuos, las 138 actividades identificadas en las 18 clases observadas. Por su parte, las variables que se tuvieron en cuenta en la identificación de correspondencias fueron los indicadores utilizados en los análisis bivariados que venimos de exponer.

A fin de establecer las asociaciones entre las modalidades de las variables y los profesores individuales, se identificó al profesor como variable ilustrativa para el análisis. Este tipo de variables no afecta los resultados del análisis ni la conformación de los ejes factoriales, pero pueden ser útiles para su caracterización (ilustración), al quedar plasmadas en el plano factorial.

\section{Descripción general de los ejes factoriales}

El análisis arrojó la presencia de tres ejes factoriales relevantes en el establecimiento de agrupaciones entre las modalidades de las variables activas consideradas. En la gráfica 11 se muestran las asociaciones resultantes; los dos primeros ejes factoriales se consideran relevantes.

5 El análisis de correspondencias múltiples (ACM) es un análisis factorial hecho sobre valores (modalidades) de variables nominales, u ordinales de pocos valores, llamadas "variables activas" del análisis. Produce una serie de gráficas en las cuales es posible identificar la contribución de cada modalidad al eje factorial por su alejamiento del centro, y la relación entre las modalidades por su cercanía espacial.
Perfiles de enseñanza según formas de interacción comunicativa en el aula. Estudio de caso con seis profesores de ciencias naturales Ángela Camargo-Uribe, Christian Hederich-Martínez

El primer factor, representado horizontalmente en la gráfica, presenta un valor propio de 0,3751 y explica el $28,86 \%$ de la inercia. El factor opone un cierto tipo de actividades de clase, ubicadas al extremo izquierdo de la gráfica, que no tienen que ver directamente con el propósito didáctico específico de una clase de ciencias con otro tipo de actividades, estas sí muy relacionadas con el tema de las clases observadas, que poseen el esquema típico de una clase escolar que busca el aprendizaje de contenidos mediante la repetición y la evaluación (véanse las modalidades del extremo derecho de la gráfica).

La tabla 4 presenta la descripción de este primer factor desde el punto de vista, tanto de las variables activas y sus modalidades como desde la consideración de las variables que lo ilustran.

Tabla 4. Descripción del factor 1 del análisis de correspondencias para las actividades de clase

\begin{tabular}{|l|l|l|l|}
\hline MODALIDADES ACTIVAS & & & \\
\hline Variable & Modalidad & Valor & N \\
\hline Tema/No tema & Otro contenido & $-10,6$ & 37 \\
\hline Tema nuevo/tema ya visto & Tema escolar & $-10,5$ & 34 \\
\hline Cercanía ciencia & No ciencia & $-10,5$ & 34 \\
\hline Uso del formato IRE & No usa IRE & $-7,5$ & 99 \\
\hline Presencia de regulación & Control & $-7,4$ & 32 \\
\hline Profesor* & Hera & $-2,5$ & 32 \\
\hline ZONA CENTRAL & & & \\
\hline Profesor* & Dionisio & 2,9 & 20 \\
\hline Tema nuevo/tema ya visto & Tema ya visto & 6,6 & 70 \\
\hline Presencia de evaluación & Evalúa & 7,2 & 36 \\
\hline Presencia de regulación & No Control & 7,4 & 106 \\
\hline Uso del formato IRE & Usa IRE & 7,5 & 39 \\
\hline Tema/No tema & $\begin{array}{l}\text { Contenido } \\
\text { didáctico }\end{array}$ & 10,6 & 101 \\
\hline
\end{tabular}

*Variables ilustrativas. No se utilizan para la conformación del factor. 
Universidad Pedagógica Nacional

Facultad de Humanidades

Gráfica 11. Ejes factoriales 1 y 2 del análisis de correspondencias con las variables que describen las actividades de clase observadas

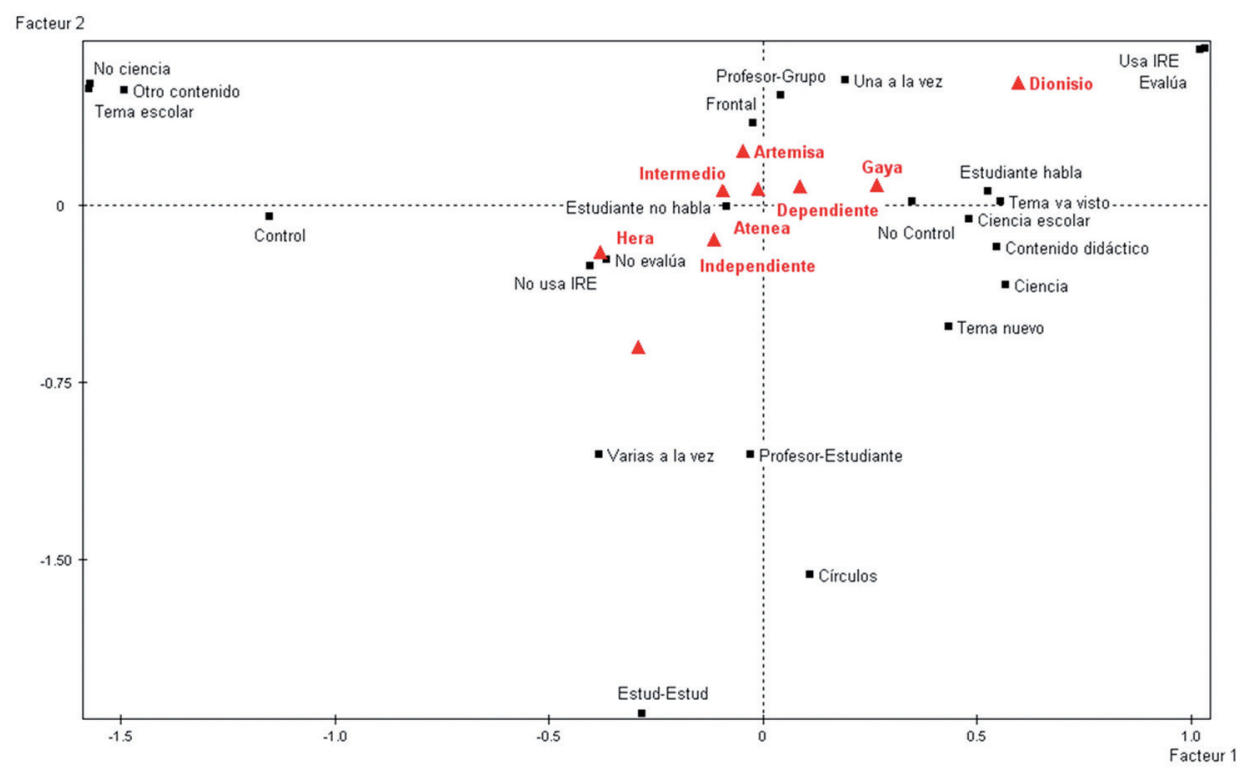

Aparentemente, lo que podría estar revelando este primer factor es el hecho de que en toda clase escolar se plantean y producen actividades que, si bien podrían tener importancia desde el punto de vista de la convivencia institucional (conversaciones sobre actividades del colegio, charlas aleccionadoras, etc.), no poseen relevancia desde el punto de vista didáctico. El hecho de que este tipo de actividad entre en contraste con un tipo de actividad escolar, esta sí de enseñanza, y que lo haga con la suficiente fuerza como para que constituyan el factor de variación con mayor peso descriptivo, es un indicador de la marcada importancia de este tipo de encuentros no didácticos en los contextos escolares de nuestro entorno social y cultural ${ }^{6}$.

Llama la atención el hecho de que este primer factor se encuentre ilustrado por la oposición entre dos profesores: Dionisio y Hera. En el extremo de las actividades que no son propiamente de enseñanza se encuentra Hera, una profesora ciertamente bastante dispuesta a ocupar tiempo de su clase a temáticas de carácter moral y a ejercer una regulación del trabajo de los estudiantes mediante un control estrecho de

6 Este hallazgo abre la puerta a estudios comparativos que consideren el nivel de importancia asignado a aspectos educativos no didácticos en entornos escolares de diferente proveniencia social o cultural. su comportamiento. En el extremo de las actividades escolares de repetición y evaluación de contenidos de la clase se encuentra Dionisio, un profesor altamente comprometido con el seguimiento de rutinas de aula para dar cuenta de contenidos de aprendizaje específicos.

A pesar de la clara definición de dos perfiles de profesores respecto de cierto tipo de actividades típicas, el problema con su postulación como perfiles de enseñanza es que los dos profesores estarían oponiéndose con base en parámetros no muy claramente comparables. En contraste afortunado, ello no ocurre con la descripción de los factores 2 y 3. Veamos.

El segundo factor del análisis, ilustrado en la dirección vertical en la gráfica 11 , posee un valor propio de 0,2523 y explica el $19,4 \%$ de la inercia. Una mirada global a la forma como las diversas modalidades quedan distribuidas en la gráfica permite identificar dos tipos de actividades que se oponen entre sí desde el punto de vista de su tenor. Un tipo de actividad que hemos llamado frontal, representada en la parte superior de la gráfica y un tipo de actividad que podría considerarse circular, representada en la parte inferior. La tabla 5 describe el factor 2 desde el punto de vista de las modalidades activas e ilustrativas que lo conforman. 
Tabla 5. Descripción del factor 2 del análisis de correspondencias para las actividades de clase

\begin{tabular}{|c|c|c|c|}
\hline VARIABLE & MODALIDAD & VALOR & $\mathbf{N}$ \\
\hline Orden temporal & Varias a la vez & $-8,7$ & 46 \\
\hline Orden espacial & Círculos & $-8,6$ & 25 \\
\hline Tipo de intercambio & Estudiante-estudiante & $-8,5$ & 14 \\
\hline Uso del formato IRE & No usa IRE & $-4,8$ & 99 \\
\hline Tipo de intercambio & Profesor-estudiante & $-4,8$ & 18 \\
\hline Profesor* & Hermes & $-2,8$ & 19 \\
\hline \multicolumn{4}{|l|}{ ZONA CENTRAL } \\
\hline Profesor* & Dionisio & 2,5 & 20 \\
\hline $\begin{array}{l}\text { Presencia de } \\
\text { evaluación }\end{array}$ & Evalúa & 4,6 & 36 \\
\hline Uso del formato IRE & Usa IRE & 4,8 & 39 \\
\hline Orden espacial & Frontal & 8,6 & 113 \\
\hline Orden temporal & Una a la vez & 8,7 & 92 \\
\hline Tipo de intercambio & Profesor-grupo & 9,9 & 106 \\
\hline
\end{tabular}

Perfiles de enseñanza según formas de interacción comunicativa en el aula. Estudio de caso con seis profesores de ciencias naturales Ángela Camargo-Uribe, Christian Hederich-Martínez

La tabla describe y configura claramente dos tipos de actividades comunicativas presentes en las clases de ciencias observadas. Por un lado están los momentos de clase en los que un cierto tema o problema está siendo trabajado por los estudiantes mediante encuentros personales, entre sí o con el profesor, y en donde no hay una intención explícita o implícita de evaluar por parte del profesor. Por otro, están las situaciones de clase en donde los encuentros comunicativos son situaciones públicas de evaluación de lo que los estudiantes ya saben mediante formatos de pregunta retórica típica del profesor.

La configuración de este factor 2 apoya la postulación, arriba señalada, de un perfil de enseñanza en el manejo interactivo del profesor de ciencias, sobre la base de la oposición frontal-circular, máxime cuando este factor se encuentra ilustrado mediante la oposición entre dos profesores. Dionisio como el profesor frontal y Hermes como el profesor circular.

Finalmente, consideraremos el factor 3 del análisis. La gráfica 12 presenta la configuración de este factor, en su relación con el factor 1, ya analizado.

Gráfica 12. Ejes factoriales 1 y 3 del análisis de correspondencias múltiples, entre las variables que describen las actividades de clase observadas

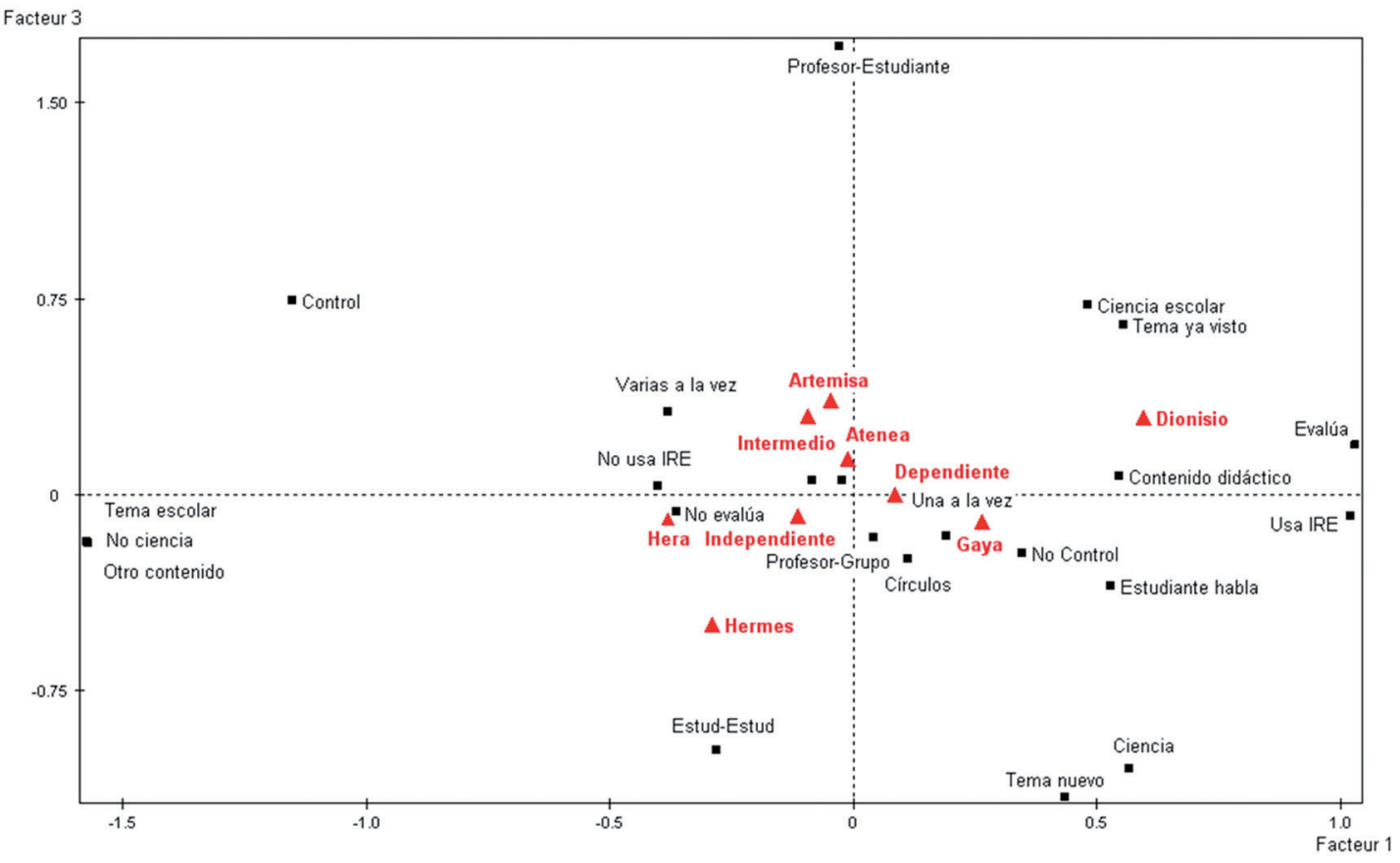


El factor 3, vertical en la gráfica 12, presenta un valor propio de 0,1902 y explica el 14,93\% de la inercia. Sobre la base de las modalidades aun no incluidas en los otros dos factores y distribuidas de acuerdo con la dirección prevista, este factor establece una oposición entre dos formas de tratamiento del tema de las ciencias naturales en las aulas observadas. En el cuadrante superior de la gráfica, se encuentran agrupadas las actividades que trabajan el tema de la ciencia desde el punto de vista propiamente escolar, con una fuerte presencia de transposiciones didácticas al contexto institucional del aula de clase. En el cuadrante inferior se ubican, en contraste, las actividades en donde se intenta replicar en el aula ciertos elementos de las condiciones de producción del conocimiento científico. La tabla 6 presenta la descripción de este factor 3, según las modalidades que lo conforman:

Tabla 6. Descripción del factor 3 del análisis de correspondencias para las actividades de clase

\begin{tabular}{|c|c|c|c|}
\hline VARIABLE & MODALIDAD & VALOR & $\mathbf{N}$ \\
\hline $\begin{array}{l}\text { Tema nuevo/tema } \\
\text { ya visto }\end{array}$ & Tema nuevo & $-7,86$ & 34 \\
\hline Cercanía ciencia & Ciencia & $-7,67$ & 39 \\
\hline Presencia de regulación & No control & $-4,55$ & 106 \\
\hline Tipo de intercambio & $\begin{array}{l}\text { Estudiante- } \\
\text { estudiante }\end{array}$ & $-4,31$ & 14 \\
\hline Tipo de intercambio & Profesor-grupo & $-2,75$ & 106 \\
\hline Presencia de guía & No guía & $-2,09$ & 103 \\
\hline Profesor* & Hermes & $-2,46$ & 19 \\
\hline \multicolumn{4}{|l|}{ ZONA CENTRAL } \\
\hline Presencia de guía & Guía & 2,09 & 35 \\
\hline Presencia de regulación & Control & 4,55 & 32 \\
\hline Tipo de intercambio & $\begin{array}{l}\text { Profesor-estudian- } \\
\text { te }\end{array}$ & 7,31 & 18 \\
\hline $\begin{array}{l}\text { Tema nuevo/tema } \\
\text { ya visto }\end{array}$ & Tema ya visto & 7,98 & 70 \\
\hline Cercanía ciencia & Ciencia escolar & 8,13 & 65 \\
\hline
\end{tabular}

Como se observa, este factor hace alusión a una dimensión de variación ya identificada en los análisis bivariados, que hemos identificado por medio de la oposición ciencia-ciencia escolar. En el extremo de la ciencia, se encuentran actividades que intentan replicar la actividad científica, con alta densidad de manejo de contenidos. En el otro extremo se encuentran las actividades en las que el tema de la ciencia se encuentra escolarizado y por lo tanto se trata de manera simple y repetida. En consonancia con lo que pudimos observar en los análisis bivariados, el primer tipo de actividad se encuentra muy presente en las clases de Hermes. El tipo de actividad opuesta parece más generalizado entre los profesores, puesto que no lo ilustra el caso de un profesor en particular.

\section{Conclusiones}

Con miras a configurar perfiles de enseñanza desde el punto de vista comunicativo, hemos presentado en este artículo el análisis comparativo de las diferentes formas como seis profesores de ciencias naturales gestionan comunicativamente sus clases. Tomando como unidad de análisis la actividad de clase, se definieron para ella dimensiones y variables descriptivas relacionadas con la forma como cada profesor asume y dirige la interacción comunicativa en sus clases.

Los análisis bivariados de preferencias (frecuencias) por ciertos tipos de interacción durante las actividades de clase permitieron la postulación de cuatro perfiles de enseñanza que pueden organizarse como oposiciones: la oposición ciencia versus ciencia escolar y la oposición frontal versus circular. El análisis de correspondencias múltiples, realizado posteriormente, corroboró y otorgó fuerza a los perfiles identificados.

Puesto que los resultados de cada tipo de análisis (bivariado y multivariado) se corroboran entre sí, los perfiles identificados poseen un sólido soporte empírico. Adicionalmente, cada una de las oposiciones corresponde a una de las dos dimensiones de análisis de situaciones comunicativas que Halliday propone en su modelo y que hemos tomado en consideración en este estudio. Mientras que la oposición ciencia versus ciencia escolar alude al campo de la situación de clase, la oposición frontal versus circular refiere directamente al tenor de esta situación. Los perfiles configurados en el análisis tienen entonces 
Perfiles de enseñanza según formas de interacción comunicativa en el aula. Estudio de caso con seis profesores de ciencias naturales Ángela Camargo-Uribe, Christian Hederich-Martínez

coherencia con el modelo teórico utilizado para la realización del análisis.

\section{¿Cómo quedarnos con una imagen clara de cada perfil?}

De todos los profesores participantes en el estudio, uno de los que presenta interacciones de aula típicas del perfil científico es Hermes. Con cierta frecuencia, Hermes realiza en sus clases simulaciones de experimentos científicos a partir de las cuales los estudiantes infieren conceptos o relaciones; las experiencias se registran y analizan sistemáticamente, de acuerdo con ciertas convenciones (informes de laboratorio) y lo construido se representa de acuerdo con procedimientos y convenciones que son parte del lenguaje de las ciencias. Todas estas actividades gestionadas por Hermes en sus clases intentan reproducir, lo más literalmente posible, la actividad científica.

Por su parte, uno de los profesores que podría ilustrar el extremo opuesto, ciencia escolar, es Atenea. Esta profesora organiza actividades de clase en las que el tema abandona el contexto de la investigación científica "pura" y sufre una transformación, para convertirse en el contenido de una actividad escolar, ya sea de reflexión analítica (comparar dos tipos de moléculas), de repetición, destinada a aprender términos nuevos (llenar espacios en blanco con las palabras adecuadas) o una actividad lúdica que permite repasar conceptos (llenar un crucigrama con términos de la jerga científica).

A su vez, para lo que corresponde a la oposición frontal versus circular, un ejemplo típico de actividad frontal es el esquema de intercambio IRE, muy frecuente en Dionisio. Como se recordará, se trata de un esquema de interacción verbal en el que el profesor realiza una pregunta, alguno de sus estudiantes la contesta y el profesor dice si la respuesta es correcta o no. Las clases de Dionisio presentan esta y otras características que lo identifican con el perfil de profesor frontal. En sus clases, el trabajo de los estudiantes es consistentemente individual, el profesor casi nunca abandona la ubicación espacial típica (de pie al frente de la clase o en algún lugar del salón en donde tiene posibilidad de supervisar a todo el grupo) y es él quien controla la totalidad de las actividades de clase.

En contraste, un ejemplo típico de actividad circular es el trabajo por grupos de estudiantes, que interactúan bajo la mirada más o menos cercana del profesor. Artemisa, una profesora con un perfil circular, organiza actividades por grupos en sus clases. Otra actividad circular que Artemisa organiza es la exposición de trabajos por parte de los estudiantes, en la que, al menos aparentemente, la responsabilidad del desarrollo de los contenidos de la clase se transfiere al estudiante, de manera tal que no todo lo que ocurre en clase resulta como consecuencia directa de la acción del profesor.

Una característica prometedora de las dos oposiciones que venimos de describir es que no se afectan entre sí. Esto significa que, desde el punto de vista de las posibilidades que tiene un profesor para la planeación y ejecución de las interacciones comunicativas en sus clases, los cuatro perfiles entran en juego. Como se observa en la tabla 7, es posible ilustrar profesores para todos los perfiles encontrados.

Tabla 7. Profesores característicos en los perfiles interactivos identificados cruzados

\begin{tabular}{c|l|l|l}
\multicolumn{2}{c|}{ PROFESOR } & \multicolumn{2}{c}{ CAMPO } \\
\multicolumn{2}{c}{ CARACTERISTICO } & CIENCIA & CIENCIA ESCOLAR \\
\hline \multirow{2}{*}{ Tenor } & Frontal & Atenea & Dionisio \\
\cline { 2 - 4 } & Circular & Hermes & Artemisa \\
\hline
\end{tabular}

Desde una perspectiva profesional, las oposiciones identificadas representan dos de las disyuntivas más difíciles a las que un profesor de educación básica o media se ve abocado diariamente. Para la oposición ciencia versus ciencia escolar, la pregunta es qué tanto y con qué tanta fidelidad reproducir en clase la actividad disciplinar que produjo el conocimiento objeto del aprendizaje. En el caso de los profesores participantes de la muestra, se trata de la investigación en ciencias naturales, pero la disyuntiva está presente también para los profesores de ciencias sociales, los profesores de artes o los profesores de literatura. En el fondo de este dilema 
Universidad Pedagógica Nacional

Facultad de Humanidades

está la pregunta por la formación en los niveles iniciales del sistema educativo.

Por su parte, para la oposición frontal versus circular, la pregunta es qué tanto y con qué tanta cercanía establecer comunicación horizontal y espontánea con los estudiantes. Responderse esa pregunta supone construirse un modelo de enseñanza con funciones y rangos de intervención específicos para cada participante de la situación pedagógica. En el fondo de esta disyuntiva está una concepción personal sobre aquello que significa ser profesor.

Por último, una mención especial merece la oposición encontrada entre actividades de clase directamente relacionadas con objetivos didácticos y actividades de clase asociada con objetivos de naturaleza más difusa, que se encontró en el factor 1 del análisis de correspondencias. Observada bajo la lente de los objetivos de este estudio, esta posible oposición didáctico versus no didáctico tendría el problema de no describir formas de enseñanza, sino más bien un parámetro que podría nominarse densidad de la enseñanza. Al no tratarse de una variable que tenga que ver con decisiones del profesor sobre formas de interacción en el aula, su análisis deberá esperar a estudios comparativos que tengan más que ver, pensamos, con la cultura escolar.

Lo anterior es indicador de la necesidad de estudios que asuman como objeto de estudio la enseñanza, tanto desde el punto de vista de cada área de formación u objetivo de aprendizaje, como de su función. A la luz de estos resultados, la función del profesor surge como un todo mucho más complejo de lo que a primera vista parece. Esperamos que este trabajo contribuya a que futuros estudios aborden este punto de manera más comprensiva.

\section{Referencias bibliográficas}

Camargo, A. (2014). Conferencistas versus conversadores. Estilos de enseñanza de profesores de ciencias y su relación con el estilo cognitivo. Revista Colombiana de Educación, 64, 273-307.
Camargo, A. (2015). El estilo de enseñanza. Una mirada comunicativa, discursiva y didáctica en el aula de Ciencias Naturales. Bogotá: Universidad Pedagógica Nacional. Colección Tesis Doctorales 7.

Cazden, C. (1988). Classroom discourse: The language of teaching and learning. Portsmouth, NH: Heinem.

Christie, F. (2002). Classroom discourse analysis. A functional perspective. Londres: Continuum.

Gumperz J. \& Hymes, D. (1972) Directions in sociolinguistics. The ethnography of communication. Nueva York: Basil Blackwell.

Halliday, M. K. A. (1982). El lenguaje como semiótica social. La interpretación social del lenguaje y del significado. México: FCE.

Kearney, P. (1984). Perceptual discrepancies in teacher communication style. Communication Education, $13,95-10$.

McCroskey, J. \& Richmond, V. (1995). Fundamentals of human communication: An interpersonal perspective. Prospect Highs, IL: Waveland.

McCroskey, J.; Richmond, V. \& McCroskey, L. (2006). An introduction to communication in the classroom. The role of communication in teaching and training. Boston: Pearson.

Mortimer, E. \& Scott, P. (2003). Meaning making in secondary science classrooms. Filadelfia: Open University Press.

Nussbaum, L. \& Tusón, A. (1996) El aula como espacio cultural y discursivo. Signos, Teoría y Práctica de la Comunicación, 17, 14-21.

Richmond, V. \& McCroskey, J. (1992). Power in the classroom: Communication, control and concern. Hillsdale, NJ: Erlbaum.

Sinclair, J. \& Coulthard, H. (1975). Towards an analysis of discourse: The English used by teachers and pupils. Londres: Oxford University Press.

Thomas, C. E.; Richmond, V. P. \& McCroskey, J. C. (1994). The association between immediacy and socio-communicative style. Communication Research Reports, 11, 107-115.

Wheeless, V. E. \& Dierks-Stewart, K. (1981). The psychometric properties of the Bern Sex-role Inventory: questions concerning reliability and validity. Communication Quarterly, 29, 173-186. 